will increase further when they will be coupled within complex algorithms, a possible starting point for future research in this field.

We also remind our colleagues that, in an attempt to increase the scientific accuracy of our review, we have used strict inclusion and exclusion criteria and therefore the number of papers that were selected for the final analyze was limited. By the time we have finalized our work, we haven't found any paper which compared the results of the Acoustic Radiation Force Impulse (ARFI) technique with the presence and development of esopha- geal varices in patients with liver cirrhosis to fulfill the inclusion criteria. We agree that ARFI, alone or in combination with Fibroscan, may bring important information regarding the progression of portal hypertension and development of esophageal varices but this statement still has to be proven in well designed prospective trials.

In this context we do believe that the original study of our Iranian colleagues, shortly presented in the letter, is interesting and we eagerly wait for the publication of the final results.

\title{
Contrast enhanced ultrasound and magnetic resonance imaging for hepatoma diagnosis
}

\section{Viroj Wiwanitkit}

DOI:

Visiting professor, Hainan Medical University, China; visiting professor, Faculty of Medicine, University of Nis, Serbia; adjunct professor, Joseph Ayobabalola University, Nigeria

\section{To the Editor,}

The recent report on contrast enhanced ultrasound (CEUS) and magnetic resonance imaging (MRI) for hepatoma diagnosis. Dumirescu et al. concluded that "CEUS can be used as the first step in the diagnosis of liver lesions, but MRI remains the gold standard diagnostic method for liver tumors [1]." In fact, CEUS was recently mentioned to be a more cost effective diagnostic tool comparing to MRI [2]. Nevertheless, the diagnostic property of both CEUS and MRI might be different in different settings. In some settings such as tropical Southeast Asia, the prevalence of liver tumor is very high and there are many varieties of tumors. The limitation in using CEUS for diagnosis of hepatoma should be men-

Received Accepted

Med Ultrason

2014, Vol. 16, No 1, 80-82

Corresponding author: Professor Viroj Wiwanitkit, M.D.

Wiwanitkit House, Bangkhae,

Bangkok Thailand

Email:wviroj@yahoo.com tioned. CEUS might be difficult to differentiate between hepatoma and cholangiocarcinoma, another common hepatobiliary tumor in Southeast Asia.

\section{References}

1. Dumitrescu CI, Gheonea IA, Sandulescu L, Surlin V, Saftoiu A, Dumitrescu D. Contrast enhanced ultrasound and magnetic resonance imaging in hepatocellular carcinoma diagnosis. Med Ultrason 2013; 15: 261-267.

2. Westwood M, Joore M, Grutters J, et al. Contrast-enhanced ultrasound using SonoVue ${ }^{\circledR}$ (sulphur hexafluoride microbubbles) compared with contrast-enhanced computed tomography and contrast-enhanced magnetic resonance imaging for the characterisation of focal liver lesions and detection of liver metastases: a systematic review and costeffectiveness analysis. Health Technol Assess 2013; 17: $1-243$.

3. Dietrich CF, Cui XW, Boozari B, Hocke M, Ignee A. Contrast-enhanced ultrasound (CEUS) in the diagnostic algorithm of hepatocellular and cholangiocellular carcinoma, comments on the AASLD guidelines. Ultraschall Med 2012; 33 Suppl 1: S57-66. 


\title{
Author's response
}

\section{Ioana-Andreea Gheonea, Larisa Sandulescu, Adrian Saftoiu}

\author{
University of Medicine and Pharmacy, Craiova
}

\section{Dear editor,}

We appreciate Prof. Viroj Wiwanitkit interest in our article "Contrast enhanced ultrasound and magnetic resonance imaging for hepatocellular carcinoma diagnosis" and we have read with interest the comments.

From results of a recent published meta-analysis, we found some reasons for which CEUS (contrast enhanced ultrasound) is considered to be the first step in the diagnosis of focal liver lesions and especially of hepatocellular carcinoma (HCC).

CEUS could provide similar diagnostic performance to contrast enhanced computer tomography (CECT) and contrast enhanced MRI (CEMRI) for the three main clinical applications: characterization of focal liver lesions (FLLs) detected on surveillance of cirrhosis patients using unenhanced ultrasound (US), detection of liver metastases in patients with known primary cancers and characterization of incidentally detected FLLs identified by unenhanced US [1].

CEUS is the most cost-effective option after inconclusive unenhanced US (compared with CECT and CEMRI). In the surveillance of cirrhosis, CEUS was found to be as effective, but $£ 379$ less costly than CECT and $£ 1063$ less costly than CEMRI [2]. Also, CEUS is time-saving, it is more accessible compared with the CECT and CEMRI and can be performed immediately after the discovery of a focal lesion on standard ultrasound with the same ultrasound system. Thus, a malignant lesion is ruled out sooner and the patient anxiety is reduced.

However, in real practice, only the FLLs that are well seen by standard ultrasound can be evaluated by CEUS. Moreover, one injection with contrast agent is necessary for every focal lesion. The performance of the ultrasound system and the experience of the physician experience can have an important impact on CEUS diagnostic accuracy also.

The study published by Vilana $\mathrm{R}$ et al showed the risk of confusing intrahepatic cholangiocarcinoma (CC) and HCC on CEUS examination because the intrahepatic cholangiocarcinomas showed in $50 \%$ of cases a similar CEUS pattern with HCC (homogeneous hyperenhancement in the arterial phase followed by homogeneous wash-out in portal or late phase). However, it should be mentioned that cholangiocarcinomas are rare in cirrhotic patients, limited to 1-2 cases/ year in reference hepatology centers [2]. A limitation of our study is that compared with others studies, we did not presented an analysis regarding the comparison between CEUS and CT/MRI in cases in which histology was the "gold-standard" method, because histology evaluation was performed on a limited number of patients $[3,4]$.

MRI with MRCP (magnetic resonance cholangiopancreatography) sequences can provide three-dimensional reconstruction of the biliary tree by using magnetic resonance technology [5]. A meta-analysis of 67 studies evaluating MRCP performance in patients with suspected biliary diseases showed an overall sensitivity of $88 \%$ and specificity of $95 \%$ [6]. MRI along with MRCP is considered the radiological modality of choice for evaluating patients with suspected cholangiocellular carcinoma, because it allows definition of the anatomy and extent of CC within the hepatobiliary system, vascular invasion, local lymphadenopathy and distant metastases [7]. Although MR features are well correlated with the pathologic changes, the appearances are nonspecific for a definitive diagnosis.

In addition, the diagnostic performance of MRI and MRCP in cholangiocellular carcinoma diagnosis was not the purpose of our study.

MRI plays an important role in assessment of liver lesions, being considered the gold standard imaging technique for HCC diagnosing, with a sensitivity of $92 \%$ in several studies [8]. In the present study, MRI correctly diagnosed the HCCs misdiagnosed by CEUS and a sensitivity of $91.4 \%$, with a specificity of $98.9 \%$ in HCC diagnosis has been found, regardless of nodules size

Therefore, we concluded in our study that the sensitivity and specificity of CEUS in diagnosing HCC was more then satisfactory, because it can be used as the first step in the diagnosis of liver lesions, but MRI remains the most confident diagnostic tool for liver tumors. 


\section{References}

1. Westwood M, Joore M, Grutters J, et al. Contrast-enhanced ultrasound using SonoVue ${ }^{\circledR}$ (sulphur hexafluoride microbubbles) compared with contrast-enhanced computed tomography and contrast-enhanced magnetic resonance imaging for the characterisation of focal liver lesions and detection of liver metastases: a systematic review and costeffectiveness analysis. Health Technol Assess. 2013;17:1243.

2. Vilana R, Forner A, Bianchi L, et al. Intrahepatic peripheral cholangiocarcinoma in cirrhosis patients may display a vascular pattern similar to hepatocellular carcinoma on contrast-enhanced ultrasound. Hepatology. 2010;51:20202029.

3. Tranquart F, Correas JM, Ladam Marcus V, et al. Real-time contrast-enhanced ultrasound in the evaluation of focal liver lesions: diagnostic efficacy and economical issues from a French multicentric study. J Radiol. 2009;90:109-122.
4. Seitz K, Bernatik T, Strobel D. et al Contrast-enhanced ultrasound (CEUS) for the characterization of focal liver lesions in clinical practice (DEGUM Multicenter Trial): CEUS vs. MRI--a prospective comparison in 269 patients. Ultraschall Med. 2010;31:492-499.

5. Romagnuolo J, Bardou M, Rahme E, et al. Magnetic resonance cholangiopancreatography: a meta-analysis of test performance in suspected biliary disease. Ann Intern Med. 2003;139:547-557

6. Schwartz LH, Coakley FV, Sun Y, et al. Neoplastic pancreaticobiliary duct obstruction: evaluation with breathhold MR cholangiopancreatography. AJR Am J Roentgenol. 1998;170:1491-1495.

7. Manfredi R, Barbaro B, Masselli G, et al. Magnetic resonance imaging of cholangiocarcinoma. Semin Liver Dis. 2004;24:155-164

8. Liu QY, Li HG, Gao M, et al. Primary clear cell carcinoma in the liver: CT and MRI findings. World J Gastroenterol 2011;17:946-952. 